\title{
Application of Bio-Image Analysis for Classification of Different Ripening Stages of Banana
}

\author{
Ganesh C. Bora ${ }^{1}$, Donqing Lin ${ }^{1}$, Pritha Bhattacharya ${ }^{1}$, Sukhwinder Kaur Bali ${ }^{2} \&$ Rohit Pathak ${ }^{1}$ \\ ${ }^{1}$ Agricultural and Biosystems Engineering, North Dakota State University, Fargo, ND, USA \\ ${ }^{2}$ Natural Resources Management, North Dakota State University, Fargo, ND, USA \\ Correspondence: Ganesh C. Bora, Agricultural and Biosystems Engineering, NDSU Dept. 7620, PO Box 6050, \\ Fargo, ND 58108-6050, USA. Tel: 1-701-231-7271. E-mail: ganesh.bora@ndsu.edu
}

Received: November 4, 2014 Accepted: December 3, 2014 Online Published: January 15, 2015

doi:10.5539/jas.v7n2p152

URL: http://dx.doi.org/10.5539/jas.v7n2p152

\begin{abstract}
Image processing is one of the important and extensively used techniques for determining physical and chemical characteristics of fruits. These characteristics are used for defining the shelf-life, packaging systems, mode of transportation and storage technique. In this method, green, yellow, and ripened banana with black spots are used for identification and classification of different ripening stages of banana. Automatic threshold separation and Aphelion 3.2 version software have been used for capturing images and analyzing structures. Hue, Saturation, and Intensity were the color coordinates used for extracting information in an image size of $451 \times 256$ pixels. Simplified histogram analysis on different color band was carried out. Result shows green band histogram can be used for classification of various ripening stages. In blue and red band histogram, it's difficult to differentiate stages.
\end{abstract}

Keywords: image processing, banana, color, pixel, ripening stages

\section{Introduction}

\subsection{General}

In agriculture, automated imaging use high resolution photography to characterize food quality. Images of individual food item is classified and analyzed based on size, shape and physical properties. Multiple entities of items ranging from 10's to 100 's of thousands of items can be measured with statistical representative distributions of item's features. Static imaging system is focused on stationary item but dynamic imaging system is capable of collecting image of items flowing through the optics. Computer vision or computer imaging is used to develop the theoretical and algorithmic techniques in which the information about the image can be extracted and analyzed. Image is taken with the camera and sends that image to the computer. In computer the other processing take place with the help of different software. As extraction of useful information from image include image acquisition, image processing and determination of correlation formula and calculation of projected area (Omid et al., 2010). Image processing techniques is having expensive kit. So for digital calculation of the product needs digital balance and caliper. Computer vision technology had been used for characterization and description of object in agriculture from many years. Soil color determination was done by Han and Hayes (1990), color vision of peach grading by Miller and Delwiche (1990) and Tarbell and Reid (1991) studied the growth and development of corn plants and developed data collection system on the basis of computer vision method.

Apples and their geometric properties are modeled by Tabatabaeefar and Rajabipour (2005). Their model shows a strong relationship between apple diameter and mass. According to Khodabandehloo (1999) surface area, mass and volume are very valuable parameter for determining sizing system. Keramat Jahromi et al. (2007) evaluated that fruits should be graded by weight rather than only size because it is more economical to provide best packing pattern on the basis of weight. But packing agricultural products by electrical mechanism on the basis of size and weight is costly and by mechanical method is full of errors. The relationship of mass, projected area and dimension developed by Tabatabaeefar et al. (2000) can be beneficial. Lorestani and Tabatabaeefar (2006) determined a mathematical model to predict the mass of kiwi fruit. In this model, dimension and projected area of the fruit were used to prefigure mass. In food technology, surface and projected area are used to ascertain the 
amount of chemical application, peeling time, and the microbial concentrations present in produce (Sabilov et al., 2002). Khojastehnazhand et al. (2009) found tape method as very time consuming and labor-intensive method and also human error prone. First the object is fully covered with tape, then the tape is removed and measured with area meter.

In recent past, image processing technique has been effectively applied for quality inspection and shape sorting. Development of imaging algorithm by Hahn and Sanchez (2000) made volume prediction of irregular shaped fruits much easier compared to conventional methods.

Omid et al. (2010) designed an image processing algorithm for citrus volume and mass. The algorithm was executed in Visual Basic programming language. They showed that citrus volume and mass are correlated to each other and design sizing systems. Panigrahi et al. (1995) applied computer vision technology for morphological characteristics of corn germplasm and studied the background segmentation and dimensional measurement. For dimensional measurement and morphological properties proper background segmentation is very important.

Bio-imaging is often integrated with particle sizing methods such as laser diffraction to validate ensemble based measurements. Applications of bio imaging include measurement of 1) texture 2) color 3) surface area or volume of non-spherical items 4) defects and 5) diseases. Currently bio-imaging technology has been widely used for quality evaluation of food products. Since quality is not a single attribute but collections of different optical features of food product. Bio-imaging technology has been recognized as one of the most important in situ technique for food quality assessment. Softening of texture and change of yellow coloration of peels are fruit ripeness indicator (Marriot et al., 1981). Texture specifies eating quality whereas peel color indicates shelf-life. Shelf-life is one of the important factors for retail distribution.

According to FAO (2002), above 67 million metric tons is produced worldwide. Usually, evenly green and matured bananas are harvested to increase the shelf life and prevent loss during transportation. They remain firm and green with no any significant physical (color and texture) and chemical changes. Consumers primarily evaluate bananas on skin color. Peel color corresponds to physical and chemical transformations during ripening process (Deullin, 1963; Wainwright \& Hughes, 1989). Commercial grading of bananas are usually performed through eye inspection and is related to change in pigment as shown in Table 1 (Le et al., 1997). Ripeness is evaluated with the help of standardized color charts. The skin color is differentiated on the basis of seven ripening stages of the color charts (Von Loesecke, 1950; Le et al., 1997). Sometimes ripeness is also estimated by instrumental techniques (Wainwright \& Hughes 1990).

Table 1. Characteristics of different grades of banana

\begin{tabular}{ll}
\hline Stage & Color \\
\hline 1 & Green \\
2 & Green and traces of yellow \\
3 & More green than yellow \\
4 & More yellow than green \\
5 & Green tip and yellow \\
6 & All yellow \\
7 & Yellow, flecked with green \\
\hline
\end{tabular}

Source: Le et al. (1997).

During ripening, first, there's a breakdown of cell walls. Due to solublization of pectic substances, the cohesion of middle lamella reduces. Second, migration of water takes place from skin to flesh, because of osmosis (Palmer, 1971; Smith et al., 1989). In case of Musa cavendish, process of starch hydrolysis and sugar synthesis completes on full ripening. But in other species of Musa, the process continues till very ripe and senescent stage.

\subsection{Objective}

The project objective is to apply image analysis technique to determine the changes in banana quality in self life (Musa domestica) with respect to change in color features, i.e., 1) RGB and 2) HSI (Hue Saturation Intensity). 


\section{Material and Methods}

\subsection{Samples of Bananas}

Mature green peel banana (Musa cavendish) was purchased from local market. It was then treated with ethylene. Uniform color and obvious defect free bananas were selected at random for image analysis in the Bio-Imaging and Sensing Center, Department of Agricultural and Biosystems Engineering, North Dakota State University. Six samples of banana were selected for each stage and there were 18 in total. Though seven stages are considered in self-life, only three stages were selected to make the proof of concept and then could be extrapolated for self-life prediction.

\subsection{Computer Vision System}

Skin color and length of the fruit is analyzed with image analysis techniques. Image processing technique was used to measure the actual projected area and surface area. In this study method (Ostu, 1979), automatic threshold separation approach has been applied to extract the banana image from plain background. Nikon camera with D3100 SLR 14.2 MP (Figure 1) and Aphelion 3.2 version, an image processing and understanding software have been used for taking images and analyzing the features.

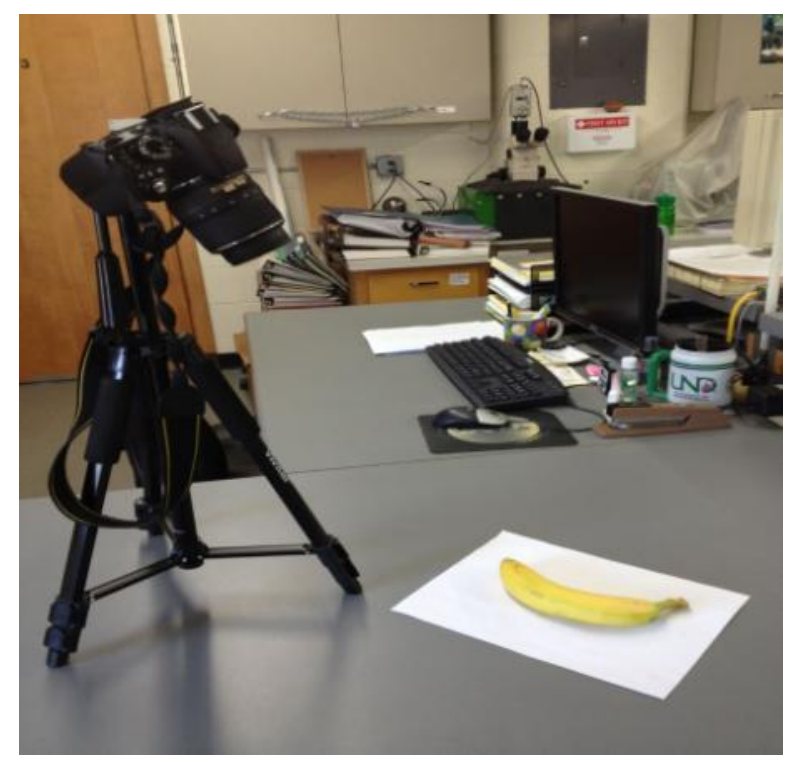

Figure 1. Nikon camera with D3100 SLR 14.2 MP

\subsection{Analysis}

Sample banana was put on a white sheet as shown in Figure 1, in longitudinal and lateral orientation. Images were taken at different stages shown in Figure 2. Calibration factor, number of pixels to linear dimension was computed from an image of $81 / 2 \times 11$ square inch of white sheet. The calibration factor was then used to calculate the axial dimensions, area and volume. The color image of banana study was carried out by computer vision system (Panigrahi \& Mishra, 1989) using D3100 SLR 14.2 MP camera. Banana was placed on a rectangular platform with white background. 


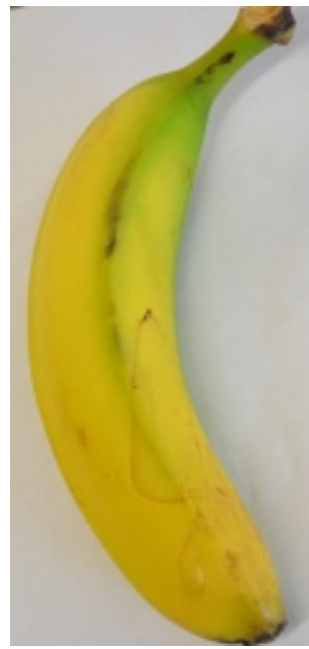

(1)

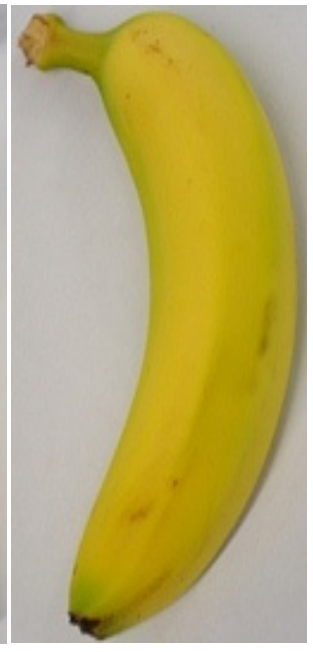

(2)

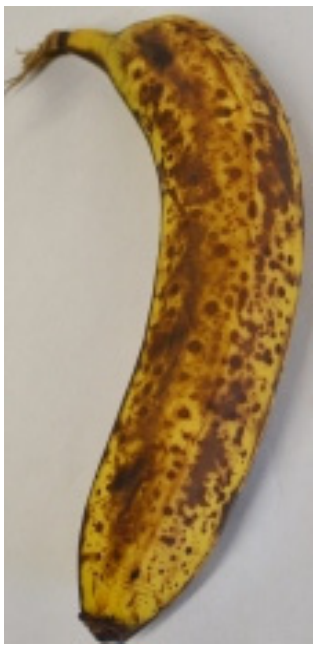

(3)

Figure 2. Changes in color and brown spot development

Hue, Saturation and Intensity (HIS) were the color coordinates used for the study of image. Each image had a size of $451 \times 256$ pixels. Hue and saturation were used to acquire color information whereas intensity for dimensional attributes. Histogram analysis was carried out on image analysis. The image area was measured in number of pixels. The number of pixels was multiplied by calibration factor to convert it into $\mathrm{mm}^{2}$. Height and width of minimum bounding rectangle (MBR) in pixels was also converted into $\mathrm{mm}^{2}$. Image of known geometry was used for the calibration of MBR. For volume measurement, calibrated area of image was multiplied with the width of other image of the same bean whose orientation was changed by $90^{\circ}$. So we can say that volume is product of area in longitudinal and thickness in lateral direction.

\subsection{Red-Green-Blue Color System}

It is hard for human eyes to detect quality differences from the minute color variations and check the quality of thousands of items. Computer imaging system has been successfully applied to acquire and process color images. The computer imaging system is composed of a color camera, capable of handling color information and color monitor that displays the information. Information is represented in term of color coordinates like Red-Green-Blue (RGB) system. The chromaticity coordinates $\mathrm{r}$, g, and $\mathrm{b}$ are represented by following equations:

$$
\begin{aligned}
& r=\frac{R}{R+G+B} \\
& g=\frac{G}{R+G+B} \\
& b=\frac{B}{R+G+B}
\end{aligned}
$$

Where: $\mathrm{R}$ is red, $\mathrm{B}$ is blue and $\mathrm{G}$ is green.

\section{Results and Discussion}

Color is most important factor for image processing and object description. As per Tri-chromatic theory, RGB color model is a combination of red, green, and blue light array. This three color component contains different value, which helps to define different color spaces. Computer images are stored in the form of matrix. These elements are referred as pixel, where there are two types of information-geometric information (pixel location in the image), and surface information (pixel intensity values). During digital image processing each red, green and blue image pixel can be represented in computer memory as binary values. These images are converted to intensities and voltages which reproduces on display. The color features which affects banana can be determined by visual image an alternative color coordinate system is HIS. HUE is the dominant spectral color or the attribute of color perception by means of which an object identified as red, green or any other specific color. Intensity (I) is the relative darkness or lightness of the dominant spectral color. Saturation(S) represents the intensity of color that shows degree of deviation from gray of the same lightness. 


\subsection{Red}

From Figure 3, it is observed that green colored banana (amplitude near about 1420 at pixel value of 198, Green) has the highest peak response than yellow colored banana (amplitude near about 1220 at pixel value of 194, Banana (1)) and ripened banana with black spots (amplitude near about 1020 at pixel value of 192, Banana (2)). Yellow colored banana peak is observed the earliest followed by ripened banana with black spots and then green banana. After reaching peak, amplitude drop-off is instant for yellow colored banana and almost similar trend in case of ripened banana with black spots. But for green banana amplitude drop-off is gradual. In case of green banana, if we follow the data population we can say that the population decreases with increase in pixel value.

For ripened banana with black spots, the density of population is almost similar throughout. However, for yellow colored banana, data population density decreases on both the sloping sides of peak point.

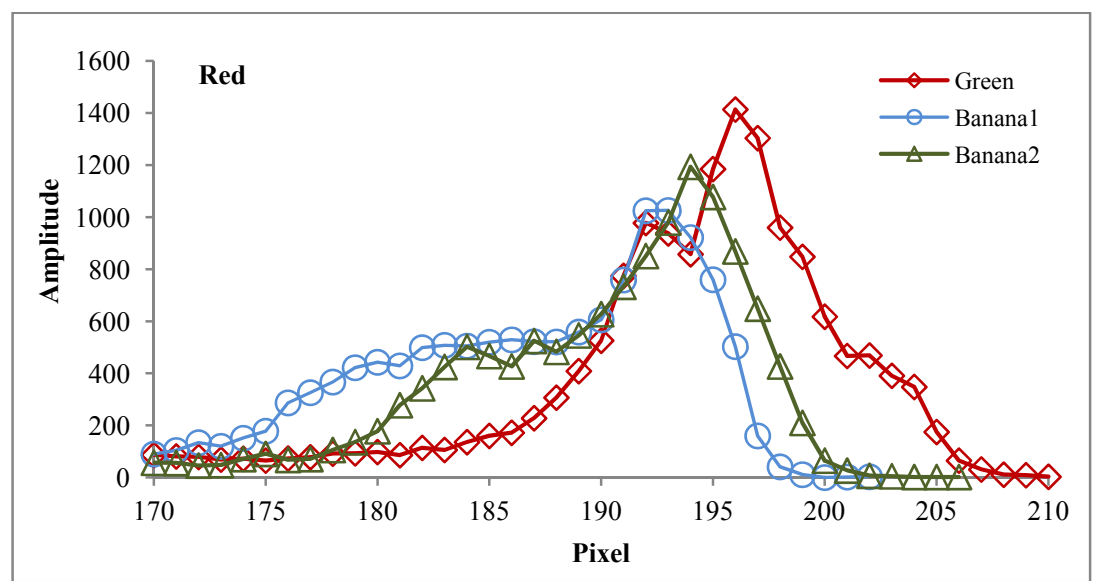

Figure 3. Pixel versus amplitude graph for three color stages of banana in RED of RGB (Banana (1) was yellow and Banana (2) was ripened banana with black spots)

We can also see that the green banana red property peak dropped off to almost 800 at 195 pixels from 1000 at 192 pixels. After that again it rises to peak point of 1420 and falls suddenly. If we follow ripened banana with black spots, we will see the graph falls sharply twice in-between 180 and 190 pixels before reaching the peak. Unlikely to green and black spot banana, yellow colored banana rises steadily to the peak without any significant rise and fall. It's plateaued at the top.

\subsection{Blue}

From Figure 4, it is observed that ripened banana with black spots (amplitude near about 990 at pixel value of 176, Banana (2)) has the highest peak response than yellow colored banana (amplitude near about 900 at pixel value of 184, Banana (1)) and green colored banana (Amplitude near about 700 at pixel value of 182, Green). Contrary to RED, here we can see ripened banana with black spots peak is observed the earliest followed by green colored and then yellow colored. 


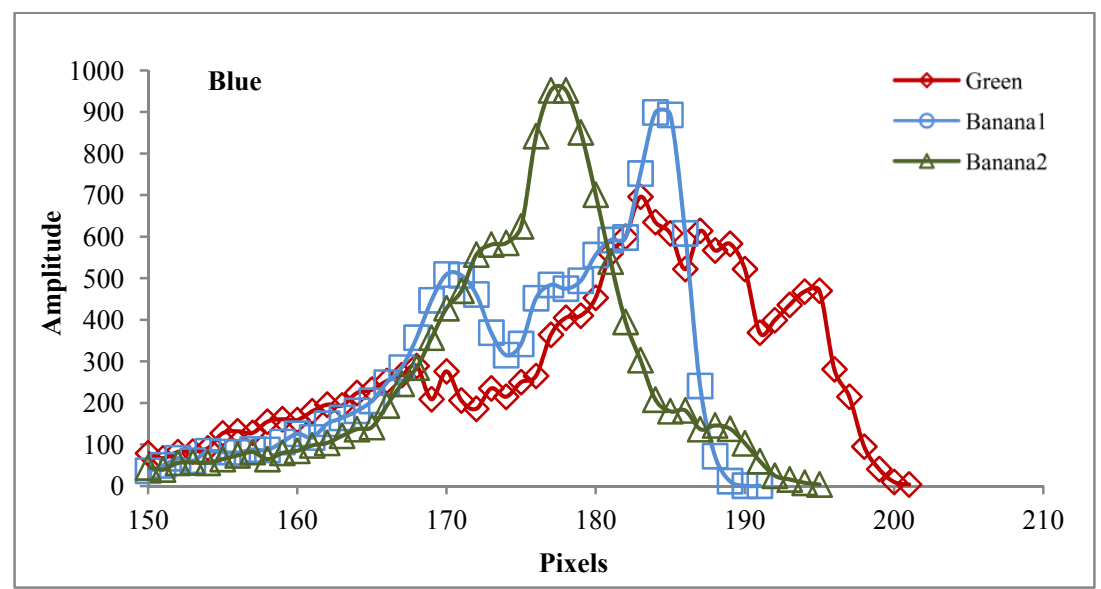

Figure 4. Pixel versus amplitude graph for three color stages of banana in BLUE of RGB (Banana (1) was yellow and Banana (2) was ripened banana with black spots)

It can also be seen from the Figure 4, green banana fluctuates wildly between 165 and 195 pixel value whereas ripened banana with black spots grows smoothly till the highest point at 176 pixel value and falls sharply. But in case of green color banana amplitude drop-off is gradual rather than sudden. Similarly, yellow colored banana shows some fluctuation at the beginning and there's a significant fall from around 500 to 300 at pixel values 170 to 175 respectively before reaching the peak and dropping suddenly. For both yellow colored and black spot banana the graph falls dramatically after it reaches the peak.

If we look at data population for ripened banana with black spots (banana-2), we can say that population is higher at beginning and end, and decreases at the rising and falling portion. Yellow colored banana shows a similar trend as banana-2. In case of green banana the density of population seems to be almost same all the way through.

\subsection{Green}

From Figure 5, it is observed that green banana (amplitude near about 1500 at pixel value of 195, Green) has the highest peak response than yellow colored banana (amplitude near about 1100 at pixel value of 189, Banana (1)) and ripened banana with black spots (amplitude near about 1050 at pixel value of 185, Banana (2)). Similar to BLUE, ripened banana with black spots peak is observed first. After that yellow colored and green colored peaks are observed second and third in turn. There's a marked fall of amplitude from around 700 to 400 at 185 and 190 pixels respectively. After that it reaches the highest point and falls back rapidly to almost zero. For yellow colored, at the beginning we can see an upward trend with some insignificant rise and fall. After reaching the peak it follows the similar trend as green banana. Ripened banana with black spots also shows an upward trend and rises to a peak value of around 1000 with slight fall at a pixel value of 185 . The declination is gradual compared to other two bananas. 


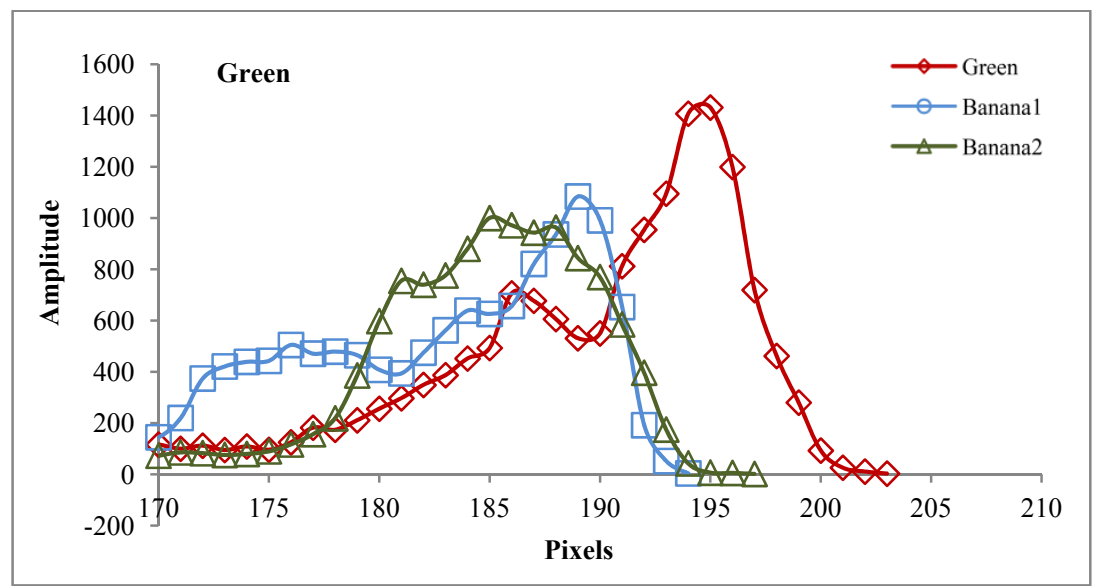

Figure 5. Pixel versus amplitude graph for three color stages of banana in GREEN of RGB (Banana (1) was yellow and Banana (2) was ripened banana with black spots)

The data population for green banana decreases with increasing pixel value. In case of ripened banana with black spots the density of population seems to be similar during the course of graph till the end. Yellow colored banana data population density decreases on both the sloping sides of peak whereas at the beginning it is denser relative to end area.

\subsection{Intensity}

From Figure 6, it is observed that green banana (amplitude near about 1200 at pixel value of 194) has the highest peak response than ripened banana with black spots (amplitude near about 1100 at pixel value of 187) and yellow colored banana (Amplitude near about 1050 at pixel value of 189). Ripened banana with black spots hit the highest point earlier than yellow and green colored banana. Green color banana attains the highest peak point The second and third highest peak point is of ripened banana with black spots and yellow colored banana respectively. After reaching the peak, steep drop off is observed for all three bananas. Black spots banana shows an upward trend until it reaches the peak point and falls slightly just before reaching peak. Yellow colored banana rises smoothly till the pixel value of 173 and then falls to amplitude of around 400 at pixel value of 180 . After this rises rapidly to peak point and decline sharply. Green colored banana also has upward trend till the topmost point with some fluctuation. It falls down markedly once from amplitude of 550 to 400 and almost flattened out to an amplitude of 625 in between the pixel value of 186 to 190.

In case of green banana, if we follow the data population we can say the population is reducing as the pixels are increasing and also the population density seems to be less at end area. In case of yellow colored banana, the density of population is near about same throughout graph except on the steeper sides where it seems to be reduced to some extent. But ripened banana with black spots, data population density is decreasing in the steeper areas else it's denser at the beginning and termination area. 


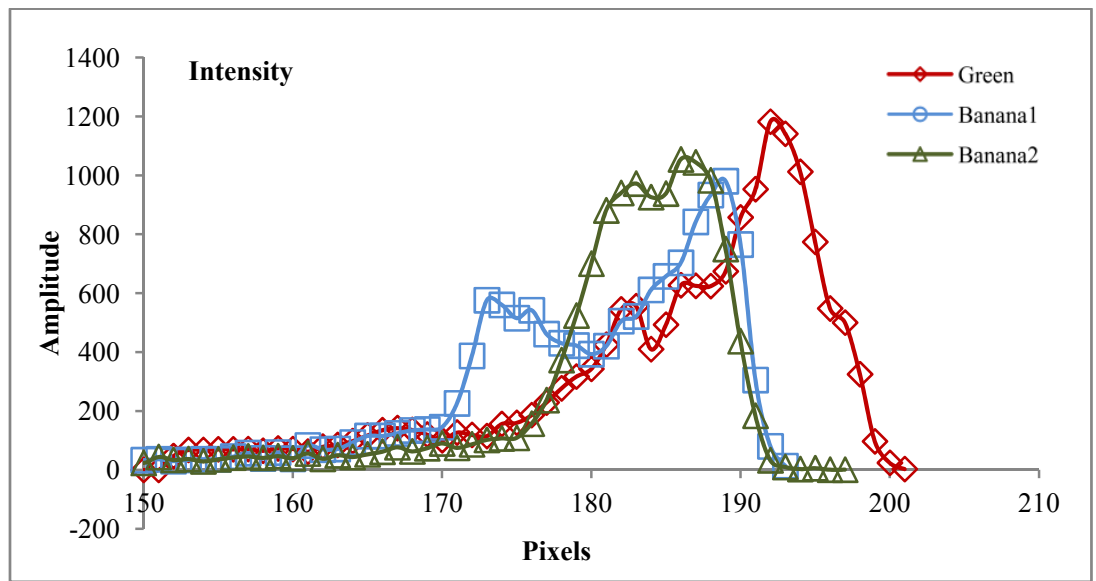

Figure 6. Pixel versus amplitude graph for three color stages of banana in INTENSITY of RGB (Banana (1) was yellow and Banana (2) was ripened banana with black spots)

\section{Conclusion}

Green band histogram can be chosen as distinguishable indicator in analyzing the banana at various stages. Histogram shapes are approximately similar as normal distribution but are skewed to the left. The decreasing pixel peaks can be used to show the banana quality in image processing system.

Blue band histogram is not recommended because the histograms are confusedly overlapped. Red band histogram looks like the green band but it's impossible to differentiate the ripe bananas. Intensity histogram is simply the average of three RGB histograms and it can be tested to compare with green band.

\section{References}

Deullin, R. (1963). Measurement of the color of the pulp of banana in preclimacteric phase. Fruit, 18, 23-26.

Food and Agriculture Organization (FAO). (2002). FAO statistical databases. FAO/United Nations. Retrieved October, 2002, from http://apps.fao.org

Hahn, F., \& Sanchez, S. (2000). Carrot volume evaluation using imaging algorithms. J. Agric. Eng. Res., 75, 243-249. http://dx.doi.org/10.1006/jaer.1999.0466

Han, Y. J., \& Hayes, J. C. (1990). Soil cover determination using color image analysis. Transactions of the ASAE, 33(4), 1402-1408. http://dx.doi.org/10.13031/2013.31486

Keramat Jahromi, M., Rafiee, S., Jafari, A., \& Tabatabaeefar, A. (2007). Determination of dimension and area properties of date (Berhi) by image analysis. International Conference on Agricultural, Food and Biological Engineering and Post-Harvest/Production Technology, KhonKaen, Thailand, 21-24 January.

Khodabandehloo, H. (1999). Physical properties of Iranian export apples (M.Sc. Thesis). Tehran University, Karaj, Iran.

Khojastehnazhand, M., Omid, M., \& Tabatabaeefar, A. (2009). Determination of orange volume and surface area using image processing technique. Int. Agrophysics, 23, 237-24.

Le, M., Slaughter, D. C., \& Thompson, J. E. (1997). Optical chlorophyll sensing system for banana ripening. Postharvest Biol. Technol., 12(3), 273-83. http://dx.doi.org/10.1016/S0925-5214 (97) 00059-8

Lorestani, A. N., \& Tabatabaeefar, A. (2006). Modeling the mass of kiwi fruit by geometrical attributes. Int. Agrophysics, 20, 135-139.

Marriot, J., Robinson, M., \& Karikari, S. K. (1981). Starch and sugar transformation during ripening of plantains and bananas. Trop. Sci., 32, 1021-1026.

Miller, B., \& Delwiche, M. (1990). A color vision system for peach grading. Transactions of the ASAE, 32(4), 1484-1490. http://dx.doi.org/10.13031/2013.31177

Omid, M., Khojasteh Nazhand, M., \& Tabatabaeifar, A. (2010). Estimating volume and mass of citrus fruits by image processing technique. J. Food Engineering, 100(2), 315-321. http://dx.doi.org/10.1016/j.jfoodeng.2010.04.015 
Palmer, J. K. (1971). The Banana. In A. C. Hulme (Ed.), The Biochemistry of Fruit and Their Products (Vol. 2, pp. 65-115). London: Academic Press.

Panigrahi, S., Mishra, M. K., Bern, C., \& Marley, S. (1995). Background segmentation and dimensional measurement of corn germplasm. Transactions of the ASAE, 38(1), 291-297. http://dx.doi.org/10.13031/2013.27841

Sabilov, C. M., Boldor, D., Keener, K. M., \& Farkas, B. E. (2002). Image processing method to determine surface area and volume of axi-symmetric agricultural products. Int. J. Food Prop., 5, 641-653. http://dx.doi.org/10.1081/JFP-120015498

Smith, N. J. S., Tucker, G. A., \& Jeger, J. (1989). Softening and cell wall changes in bananas and plantains. Aspects Appl. Biol., 20, 57-65.

Tabatabaeefar, A., \& Rajabipour, A. (2005). Modeling the mass of apples by geometrical attributes. Sci. Hortic., 105, 373-382. http://dx.doi.org/10.1016/j.scienta.2005.01.030

Tabatabaeefar, A., Vefagh-Nematolahee, A., \& Rajabipour, A. (2000). Modeling of orange mass based on dimensions. J. Agr. Sci. Tech., 2, 299-305.

Tarbell, K. A., \& Reid, J. F. (1991). A computer vision system for characterizing corn growth and development. Transactions of the ASAE, 34(5), 2245-2255. http://dx.doi.org/10.13031/2013.31864

Von Loesecke, H. W. (1950). Bananas - Chemistry, physiology, technology. Interscience Publishers, New York.

Wainwright, H., \& Hughes, P. (1989). Objective measurement of banana pulp color. Int. J. Food Sci. \& Technol., 24, 553-558. http://dx.doi.org/10.1111/j.1365-2621.1989.tb00679.x

Wainwright, H., \& Hughes, P. (1990). Changes in banana pulp color during ripening. Fruits, 45(1), 25-28.

\section{Copyrights}

Copyright for this article is retained by the author(s), with first publication rights granted to the journal.

This is an open-access article distributed under the terms and conditions of the Creative Commons Attribution license (http://creativecommons.org/licenses/by/3.0/). 